\title{
CARACTERIZAÇÃO E USOS DO \\ PARQUE FLORESTAL QUEDAS DO RIO BONITO \\ LOCALIZADO NA CIDADE DE LAVRAS/MG - PESQUISA DE OPINIÃO -
}

\author{
LARISSA MARTINIANO DE CARVALHO ${ }^{1}$ \\ PATRÍCIA DUARTE DE OLIVEIRA PAIVA ${ }^{2}$ \\ FAUSTO WEIMAR ACERBI JUNIOR ${ }^{3}$ \\ SILVÉRIO JOSÉ COELHO ${ }^{2}$ \\ FERNANDA CRISTIANE SIMÕES ${ }^{4}$
}

\begin{abstract}
RESUMO - O Parque Quedas do Rio Bonito é o único parque metropolitano da cidade de Lavras - Minas Gerais. Localizado fora do perímetro urbano, possui, como principal objetivo, a preservação ambiental. É, ainda, muito utilizado como atração turística, possuindo grande importância para a cidade. Com a finalidade de fazer uma análise da visão da população sobre suas características e uso, foi realizada uma pesquisa de opinião, por meio de entrevistas, na qual foram amostrados representantes de 600 famílias, aplicando-se um questionário
\end{abstract}

TERMOS PARA INDEXAÇÃO: Parques, lazer. padronizado com perguntas diretas e descritivas. Pelos resultados, verificou-se que o Parque Quedas do Rio Bonito é frequientado por $60,5 \%$ dos entrevistados, sendo esses principalmente das classes de renda média e alta. Observou-se ainda que $39,5 \%$ dos entrevistados nunca visitaram o parque e o principal motivo alegado foi a falta de condução. Verificou-se que o parque atrai parte da população, mesmo estando fora do perímetro urbano, pois possui atividades e contemplação diversificadas.

\section{CHARACTERIZATION AND USES OF THE FOREST PARK "FALLS OF RIO BONITO" LOCALIZED IN THE MUNICIPALITY OF LAVRAS/MG, THROUGH A OPINION RESEARCH}

\begin{abstract}
Falls of Rio Bonito" is the unique metropolitan park of the Lavras city - Minas Gerais state. It is localized out of the urban perimeter, and it has as main objective the environment preservation. It is still very used as an touristic attraction, which is very important for the city. With the purpose of doing an analysis of the population vision on their characteristics and use, we realized an opinion research was accomplished, by interviewing representatives of 600 families, to them a standardized questionnaire was
\end{abstract}

applied, with direct and descriptive questions. The results showed that the Park "Falls of Rio Bonito" is frequented by $60.5 \%$ of the interviewed, being these mainly of the classes of medium and high income. It was observed that $39.5 \%$ of the interviewed had never visited the park and the main alleged reason was the transport lack. It was verified that the park attracts part of the population in spite of being out of the urban perimeter, because it has activities and contemplations diversified.

INDEX TERMS: Parks, leisure.

\footnotetext{
1. Engenheiro Agrônomo, Msc. Fitotecnia.

2. Professores do Departamento de Agricultura, UNIVERSIDADE FEDERAL DE LAVRAS/UFLA, Caixa Postal 37 37200-000 - Lavras, MG.

3. Professor do Departamento de Engenharia Florestal/UFLA

4. Doutoranda, Departamento de Agricultura/UFLA.
} 


\section{INTRODUÇÃO}

Parques são grandes espaços abertos livres, urbanos ou entre cidades, arborizados, podendo conter áreas de vegetação natural e áreas de vegetação plantada. Possuem finalidades de recreação, lazer e conservação da natureza, sendo de grande importância para a saúde física e mental das populações urbanas. Do ponto de vista ecológico, contribuem para a proteção da flora, da fauna, da água e do solo, exercendo efeito benéfico para o microclima (DEMATTÊ, 1999).

A cidade de Lavras possui um parque de propriedade particular localizado fora do perímetro urbano, denominado Parque Quedas do Rio Bonito, com área aproximada de 70 ha, tendo como objetivo principal preservar importantes sistemas de valores naturais e culturais, protegendo recursos genéticos, desenvolvendo a educação ambiental e oferecendo oportunidades para a recreação, além de permitir a realização de pesquisas de caráter científico.

O Parque Florestal Quedas do Rio Bonito é mantido pela Fundação Abraham Kasinski e foi criado em uma área de onde era retirada a água que abastecia a cidade. Ainda restam no local construções da época (UFLA, 2002).

Em relação à estrutura, o Parque possui um espaço de convívio de uso múltiplo, com restaurante, centro de informações, administração, playground, anfiteatro e recantos para descanso. Para esportes e recreação, possui quadras poliesportivas e vestiários, além de áreas para caminhadas. Possui, ainda, um mirante, além de quedas de água e poços naturais, situados próximo à cachoeira do "Poço Bonito", que dá nome ao parque.

Por se tratar de um parque muito importante para a cidade de Lavras, realizou-se esta pesquisa com a finalidade de fazer uma análise da situação atual, visando às suas características e uso, de acordo com a visão da população.

\section{METODOLOGIA}

O trabalho foi desenvolvido no município de Lavras, que se localiza a $21^{\circ} 14$ ' 30 " latitude S e $45^{\circ} 00^{\prime} 10$ " longitude $\mathrm{W}$, na região sul de Minas Gerais. O clima é mesotérmico, com verões brandos e chuvosos.

A pesquisa foi feita diretamente com o entrevistado, que respondeu a um questionário padronizado com questões diretas e descritivas. Para o tamanho da amostra, foi considerada uma margem de erro de $4,1 \%$, com coeficiente de confiança de $95 \%$. Dessa forma, o tamanho da amostra foi determinado pela fórmula estatística (BEARZOTI e OLIVEIRA, 1997):

$$
\mathrm{e}=\mathrm{Z}_{\alpha / 2} \cdot \sqrt{\frac{p \cdot q}{n}}
$$

Sendo:

- e: erro padrão da proporção;

- $Z_{\alpha / 2}$ : é um valor da Tabela de $\mathrm{Z}$, referente ao grau de 95\% de confiança;

- p: proporção dos indivíduos que possuem a característica de interesse;

- q: proporção dos indivíduos que não possuem a característica de interesse, sendo determinado por $\mathrm{q}=$ $1-\mathrm{p}$

- n: é o tamanho da amostra.

Assim, o tamanho determinado para a amostra foi:

$$
\mathrm{e}=\mathrm{Z}_{\alpha / 2} \cdot \sqrt{\frac{p \cdot q}{n}}
$$

Para:

Grau de confiança $=95 \% \rightarrow \mathrm{Z}_{1-\alpha / 2}=\mathrm{Z}_{0,975}=1,96 \rightarrow \alpha$ $=0,05$

$\mathrm{p}=0,50$

$\mathrm{q}=1-\mathrm{p} \rightarrow \mathrm{q}=1-0,5=0,5$ então $\mathrm{q}=0,5$ (como não se tem um conhecimento a priori com relação à proporção de pessoas que possuem a característica de interesse, adota-se o valor de $\mathrm{p}=\mathrm{q}=0,5$ )

Dessa forma:

$$
\begin{array}{ll}
e=1,96 \frac{\sqrt{0,5 \cdot 0,5}}{\sqrt{n}}= & 1,96 \frac{\sqrt{0,25}}{\sqrt{n}}=1,96 \frac{\sqrt{1}}{\sqrt{4 n}}= \\
1,96 \frac{1}{4 \sqrt{n}}= & e=\frac{1}{\sqrt{n}}
\end{array}
$$

Considerando-se e $=0,041(4,1 \%)$

$$
\begin{gathered}
0,041 \cong \frac{1}{\sqrt{n}} \quad n \cong \frac{1}{0,001681} \cong 594,88 \\
\mathrm{n} \cong 600,00 \text { pessoas }
\end{gathered}
$$

Dessa forma, o tamanho da amostra (n) foi de 600 pessoas.

Determinado o tamanho da amostra, realizou-se a amostragem dividindo-se o mapa da cidade em 21 setores residenciais. O tamanho da amostra de cada setor 
foi proporcional ao número de pessoas residentes em cada setor (Tabela 1).

$\mathrm{O}$ sorteio das unidades amostrais seguiu o sistema sistematizado, com salto, entre unidades amostrais, de 20 residências.
Antes de o questionário ser utilizado de forma definitiva, ele foi submetido a um pré-teste, conforme determinação de Marconi (1996), a fim de detectar possíveis falhas existentes. O questionário utilizado está descrito a seguir:

TABELA 1 - Setores da cidade de Lavras/MG com seus respectivos bairros e tamanho da amostra para a realização da pesquisa de campo, 2000.

\begin{tabular}{|c|c|c|c|}
\hline Setor & Bairro & Freqüência & $\begin{array}{c}\% \text { da } \\
\text { amostra }\end{array}$ \\
\hline 1 & Fabril e Esplanada & 18 & 3,0 \\
\hline 2 & Centro baixo & 30 & 5,0 \\
\hline 3 & Centro Alto e Jardim das Palmeiras & 36 & 6,0 \\
\hline 4 & Ipês e Inácio Valentine & 18 & 3,0 \\
\hline 5 & Nilton Teixeira, José Moura de Amaral, Vila Rosalina e Jardim das Acácias. & 30 & 5,0 \\
\hline 6 & Belizandra e Dona Flor & 24 & 4,0 \\
\hline 7 & Murad, Serra Azul, Dona Julieta, Vila Joaquim de Sales e São Vicente & 12 & 2,0 \\
\hline 8 & Vila Cruzeiro do Sul, José Vilela, Ouro Preto e Vila Vera Cruz & 36 & 6,0 \\
\hline 9 & Mutirão e João da Cruz Botrel & 12 & 2,0 \\
\hline 10 & Vila São Sebastião, Vila Santa Terezinha, Retiro e Jardim São Paulo, & 30 & 5,0 \\
\hline 11 & $\begin{array}{l}\text { Centenário, Padre Dehon, Nossa Senhora Aparecida, Vila Brasília, Cond. Al- } \\
\text { deia de Sagres e Presidente Kennedy }\end{array}$ & 24 & 4,0 \\
\hline 12 & $\begin{array}{l}\text { Nova Lavras, Vila Ester, Jardim das Alterosas, Vila Bandeirante, Jd. América, } \\
\text { Olaria, Belo Horizonte, Vila Pitangui, Monte Líbano I e II, Eldorado e Nossa } \\
\text { Senhora do Líbano }\end{array}$ & 42 & 7,0 \\
\hline 13 & Vila Mariana, Júlio Pinto, Vila Paraíso e Jardim Europa & 30 & 5,0 \\
\hline 14 & Lavrinhas, Nossa Senhora de Lourdes e Aquenta Sol & 30 & 5,0 \\
\hline 15 & São Vicente, Vila Joaquim Sales, Dona Julieta e Serra Azul. & 36 & 6,0 \\
\hline 16 & $\begin{array}{l}\text { Vila Martins, Planalto, Cascalho, Vila Alzira, Jardim Vila Rica, Santa Filome- } \\
\text { na e Bairro de Fátima. }\end{array}$ & 36 & 6,0 \\
\hline 17 & Água Limpa, e Nova Água Limpa & 24 & 4,0 \\
\hline 18 & $\begin{array}{l}\text { Santa Efigênia, Parque da Bocaina, Dr. Paulo Menicucci, Pedro Silvestre, Dis- } \\
\text { trito Industrial, Jardim Bela Vista, Jardim das Magnólias, Vale do Sol, Aero- } \\
\text { porto e Samauma }\end{array}$ & 30 & 5,0 \\
\hline 19 & Jardim Floresta, Costa Pinto, Bicame, Artur Bernardes e Dr. João Ribeiro & 36 & 6,0 \\
\hline 20 & Jardim Glória, Jardim Campestre e Cond. Flamboyants & 42 & 7,0 \\
\hline 21 & Vila São Francisco & 24 & 4,0 \\
\hline Total & & 600 & 100,0 \\
\hline
\end{tabular}

Fonte: MDA Pesquisa de Opinião Pública e Consultoria Estatística LTDA 


\section{QUESTIONÁRIO PARA AVALIAÇÃO DA CARACTERIZAÇÃO E USOS DO PARQUE QUEDAS DO RIO BONITO - LAVRAS/ MG}

01. Setor:

02. Qual o seu nível de escolaridade? 1. até Ensino Fundamental/ 2. Ensino Médio/ 3. Superior

03. Qual a renda familiar mensal? 1. $<\mathrm{R} \$ 300,00 / 2$.

$\mathrm{R} \$ 300,00$ a $\mathrm{R} \$ 1.500,00 / 3 .>\mathrm{R} \$ 1.500,00$

04. Você conhece o Parque Municipal Florestal Abraham Kasinski (Poço Bonito)? 1. Não Conheço (ir para 5)/ 2. Já ouvi falar, mas nunca fui (ir para 5)/ 3. Conheço, já visitei (ir para 06)

05. Se não conhece, por que você nunca foi? (Múltiplas respostas)

06. Se conhece, costuma visitar o Parque com que freqüência? 1. Uma vez por semana (ir para a 08)/ 2. Uma vez a cada 15 dias (ir para a 08)/ 3 . Uma vez por mês (ir para a 08)/ 4. Não tenho frequiência (ir para a 08) /5. Só fui uma vez e não voltei mais ( ir para 07).

07. Por que deixou de freqüentar? (Múltiplas respostas) 08. O que mais the atraiu quando visitou este parque? (Múltiplas respostas) 1. Cachoeira/ 2. Trilha / 3. Paisagem/ 4. Lago/ 5. Vegetação/ 6. As pessoas/ 7. O fato de estar distante da cidade/ 8. Os animais silvestres/ 9. O contato com a natureza/ 10 . Outros.

A análise dos dados foi feita pelo software SPSS, no qual foram obtidas as freqüências percentuais (BEARZOTI e OLIVEIRA, 1997).

\section{RESULTADOS}

Os 21 setores onde foram realizadas as entrevistas da pesquisa de campo foram listados na Tabela 1. Nota-se que os setores Nova Lavras e Jardim Glória são os mais populosos e, em conseqüência, apresentam uma maior porcentagem de entrevistados.

Quanto ao nível de escolaridade, a maioria dos entrevistados $(62,2 \%)$ possuía o Ensino Fundamental (Tabela 2); esses números aproximam-se das médias de escolaridade do município.

TABELA 2 - Nível de escolaridade dos entrevistados. Lavras/MG, 2001.

\begin{tabular}{lc}
\hline \multicolumn{1}{c}{ Grau de escolaridade } & Percentual \\
\hline Até Ensino Fundamental & 62,2 \\
Ensino Médio & 23,7 \\
Superior & 14,2 \\
\hline Total & 100,0 \\
\hline
\end{tabular}

Para classificação da renda familiar, utilizou-se o critério de classes de renda baseadas no salário mínimo (conforme MDA Pesquisa de Opinião Pública e Consultoria Estatística Ltda). A classe de renda baixa possui renda de até dois salários mínimos; a classe de renda média, de dois a dez salários e para classe de renda alta, maior de dez salários.

A classe de renda predominante na cidade é a média, com $55,6 \%$ da população (comunicação pessoal, MDA - Pesquisa de Opinião Pública e Consultoria Estatística Ltda). Na pesquisa, identificou-se que $51,3 \%$ dos entrevistados pertenciam a essa categoria (Tabela 3).

TABELA 3 - Renda familiar mensal das famílias entrevistadas. Lavras/MG, 2001 (obs.: valor do salário mínimo vigente na época da pesquisa: $\mathrm{R} \$ 151,00$ ).

\begin{tabular}{lc}
\hline \multicolumn{1}{c}{ Renda familiar mensal } & Percentual \\
\hline$<\mathrm{R} \$ 300,00$ (classe de renda baixa) & 25,0 \\
$\mathrm{R} \$ 300,00$ a $\mathrm{R} \$ 1.500,00$ (classe de ren- & 51,3 \\
da média) & 23,7 \\
$>1.500,00$ (classe de renda alta) & 100,0 \\
\hline Total & \\
\hline
\end{tabular}

Objetivando-se avaliar o conhecimento da existência e a utilização do Parque pelos moradores da cidade de Lavras, perguntou-se aos entrevistados se já o haviam visitado. Dos entrevistados, $60,5 \%$ responderam que sim, enquanto $39,5 \%$ disseram que não. Desses últimos, 3\% desconheciam a sua existência (Tabela 4).

TABELA 4 - Porcentagem dos entrevistados que conheciam o Parque Quedas do Rio Bonito. Lavras/MG, 2001.

\begin{tabular}{lc}
\hline \multicolumn{1}{c}{ Resposta } & Percentual \\
\hline Conhece e já visitou & 60,5 \\
Já ouviu falar, mas nunca visitou & 36,5 \\
Não sabia da existência do parque & 3,0 \\
\hline Total & 100,0 \\
\hline
\end{tabular}

Dos que ouviram falar sobre o parque, mas nunca foram, 38,4\% pertencem à classe de baixa renda; $50,2 \%$ pertencem à classe de renda média e $11,4 \%$ pertencem à classe de renda alta (Tabela 5). 
TABELA 5 - Porcentagem dos entrevistados em relação ao conhecimento sobre o Parque Quedas do Rio Bonito, por renda familiar. Lavras/MG, 2001.

\begin{tabular}{lcccc}
\hline \multirow{2}{*}{ Respostas } & \multicolumn{3}{c}{ Renda R\$ } & Total \\
\cline { 2 - 5 } & $\begin{array}{c}\mathbf{3 0 0 , 0 0} \\
(\mathbf{\%})\end{array}$ & $\begin{array}{c}\mathbf{3 0 0 , 0 0} \mathbf{a} \mathbf{1 . 5 0 0 , 0 0} \\
(\mathbf{\%})\end{array}$ & $\begin{array}{c}>\mathbf{1 . 5 0 0} \\
(\mathbf{\%})\end{array}$ & $(\boldsymbol{\%})$ \\
\hline - Não sabia da existência & 38,9 & 27,8 & 33,3 & 3,0 \\
- Já ouviu falar, mas nunca visitou & 38,4 & 50,2 & 11,4 & 36,5 \\
- Conhece e já visitou & 16,2 & 53,2 & 30,6 & 60,5 \\
\hline
\end{tabular}

Em relação à classe de renda dos entrevistados freqüentadores do Parque, pode-se observar que a maioria deles pertence às classes de renda alta $(30,6 \%)$ e média $(53,2 \%)$, contra $16,2 \%$ da classe de renda baixa (Tabela 6). O fato de a maioria dos freqüentadores pertencerem à classe média e alta pode ser justificado por se tratar de um parque particular.

TABELA 6 - Renda familiar mensal das famílias freqüentadoras do Parque Quedas do Rio Bonito. Lavras/MG, 2001 (obs.: Valor do salário mínimo vigente: $\mathrm{R} \$ 151,00)$.

\begin{tabular}{lc}
\hline \multicolumn{1}{c}{ Renda familiar mensal } & Percentual \\
\hline$<\mathrm{R} \$ 300,00$ (classe de renda baixa) & 16,2 \\
$\mathrm{R} \$ 300,00$ a R\$ 1.500,00 (classe de & 53,2 \\
renda média) & 30,6 \\
$>1.500,00$ (classe de renda alta) & 100,0 \\
\hline Total
\end{tabular}

Os motivos alegados pelos entrevistados por nunca terem visitado o parque foram: falta de condução $(40,2 \%)$; falta de oportunidade $(34,7 \%)$; falta de interesse/motivação $(21,9 \%)$ e o fato de ter que pagar um ingresso $(16,4 \%)$ Tabela 7.

Observou-se que o principal fator limitante à visitação do parque está relacionado à falta de condução. Esse problema poderia ser solucionado com a colocação de uma linha de ônibus específica para facilitar o acesso das pessoas.

Verificou-se que grande parte dos entrevistados que nunca visitaram o Parque Quedas do Rio Bonito pertencem às classes de renda baixa e média, sendo essas as famílias mais prejudicadas quando há necessidade de meio de transporte para deslocamento. Isso também pode ser constatado na Tabela 7, quando se analisam os motivos pelos quais os entrevistados não conhecem o parque, relacionando-os com sua renda. Verifica-se que, para os entrevistados de classe de renda baixa e média, o principal motivo da não visitação é a falta de um transporte público. Para os não-freqüentadores de classe de renda alta, o problema é a falta de oportunidade e tempo.

$\mathrm{O}$ fato de ser um parque que, a princípio, chegou a cobrar ingressos para a sua visitação e hoje cobra-se uma taxa somente para a utilização da cachoeira, provocou a não-visitação de parte dos entrevistados pertencentes à classe de renda baixa, sendo esse também um importante fator limitante (Tabela 7). A cobrança de ingresso, no entanto, é fundamental para a manutenção da área da cachoeira.

Considerando a freqüência de visitas ao parque pelas famílias que já o visitaram pelo menos uma vez, verificou-se que $51,2 \%$ não têm uma freqüência definida e $42,2 \%$ só visitaram o parque uma vez e não voltaram mais (Tabela 8). 
TABELA 7 - Motivos alegados pelos entrevistados que nunca visitaram o Parque Quedas do Rio Bonito, de acordo com a renda familiar (múltiplas respostas). Lavras/ MG, 2001.

\begin{tabular}{lcccc}
\hline \multicolumn{1}{c}{ Motivos } & $\begin{array}{c}<\mathbf{3 0 0 , 0 0} \\
(\mathbf{\%})\end{array}$ & $\begin{array}{c}\mathbf{3 0 0 , 0 0} \mathbf{a} \mathbf{1 . 5 0 0 , 0 0} \\
(\mathbf{\%})\end{array}$ & $>\mathbf{1 . 5 0 0}(\boldsymbol{\%})$ & Geral \\
\hline Falta de condução & 44,0 & 43,6 & 12,0 & 40,2 \\
Falta de oportunidade & 33,3 & 33,6 & 44,0 & 34,7 \\
Não têm motivação/interesse & 17,9 & 23,6 & 28,0 & 21,9 \\
Têm que pagar ingresso & 23,8 & 13,6 & 4,0 & 16,4 \\
Falta tempo & 13,1 & 15,5 & 20,0 & 15,1 \\
É muito longe & 16,7 & 15,5 & 4,0 & 14,6 \\
Falta de hábito de sair & 6,0 & 4,5 & 4,0 & 5,0 \\
Têm outras opções para sair & - & 3,6 & 8,0 & 2,7 \\
Não gostam de parques & 1,2 & 3,6 & - & 2,3 \\
Outros & 10,7 & 10,0 & 16,0 & 11,0 \\
Não sabem/Não responderam & 1,2 & - & - & 0,5 \\
\hline
\end{tabular}

(Base de dados referente aos 219 entrevistados [36,5\%]que nunca visitaram o Parque Quedas do Rio Bonito).

TABELA 8 - Porcentagem dos entrevistados que visitam o Parque Quedas do Rio Bonito, de acordo com a freqüência de visitas. Lavras/MG, 2001.

\begin{tabular}{lc}
\hline \multicolumn{1}{c}{ Respostas } & Percentual \\
\hline Só foram uma vez e não voltaram mais & 42,2 \\
Não têm freqüência definida & 51,2 \\
Uma vez por mês & 4,4 \\
15 em 15 dias & 1,9 \\
Uma vez por semana & 0,3 \\
\hline Total & 100,0 \\
\hline
\end{tabular}

(Base de dados referente aos 363 entrevistados [60,5\%] que visitaram o Parque Quedas do Rio Bonito, independente de sua frequiência).

Os principais motivos apresentados pelos entrevistados que visitaram o parque apenas uma vez e não retornaram estão listados na Tabela 9. Entre esses, destacaram-se a falta de condução para $30,1 \%$ dos entrevistados, a distância e a falta de tempo para $23,5 \%$, e o fato de ter que pagar ingresso para $17,6 \%$.
TABELA 9 - Motivos apresentados para o fato de só terem visitado o parque Quedas do Rio Bonito uma vez (múltiplas respostas). Lavras/MG, 2001.

\begin{tabular}{lr}
\hline \multicolumn{1}{c}{ Respostas } & Percentual \\
\hline Falta de condução & 30,1 \\
É muito longe & 23,5 \\
Falta tempo & 23,5 \\
É caro/têm que pagar ingresso & 17,6 \\
Falta de oportunidade & 17,0 \\
Têm outras opções para sair & 12,4 \\
Não têm motivação & 9,2 \\
A reforma acabou com o parque & 3,9 \\
Não pode levar alimentação & 2,6 \\
Falta segurança & 2,6 \\
Não gostam do parque & 2,0 \\
Falta divulgação & 2,0 \\
Não tem novidades/é sempre igual & 1,3 \\
Horário de funcionamento inadequado & 0,7 \\
Não têm hábito & 0,7 \\
Outros & 11,8 \\
Não sabem/não quiseram responder & 1,3 \\
\hline
\end{tabular}

(Base de dados referente aos 153 entrevistados $[42,2 \%]$ que visitaram o parque apenas uma vez). 
Observou-se, também, que alguns dos entrevistados citaram o fato de "não poder levar alimentação", pois o parque exige que seus freqüentadores consumam alimentos do local. Isso foi justificado por um funcionário, que revelou que a limpeza do parque não era mantida pelos visitantes. Essa declaração mostra a necessidade de um trabalho de educação ambiental junto aos visitantes, além de, com isso, o parque obrigar o visitante a consumir alimentos do local, justificando mais uma vez a baixa presença de visitantes de baixa renda.

Perguntou-se, também, aos entrevistados sobre as possibilidades de lazer que o Parque oferece e, entre essas, quais mais agradavam. As respostas estão descritas na Tabela 10. Observa-se que as atividades mais valorizadas pelos entrevistados foram: contato com a natureza $(74,7 \%)$, a paisagem $(60,6 \%)$, a cachoeira $(51,2 \%)$, a trilha $(39,9)$ e a vegetação $(21,5 \%)$.

$\mathrm{Na}$ análise das respostas, pode-se averiguar que o local parece se tratar de um parque com atividades e contemplação diversificadas. Por isso, atrai a população, mesmo localizado fora do perímetro urbano.

TABELA 10 - Atividades mais atrativas proporcionadas pelo Parque Quedas do Rio Bonito (múltiplas respostas). Lavras/MG, 2001.

\begin{tabular}{lc}
\hline \multicolumn{1}{c}{ Atividades } & Percentual \\
\hline Contato com a natureza & 74,7 \\
Paisagem & 60,6 \\
Cachoeira & 51,2 \\
Trilha & 39,9 \\
Vegetação & 21,5 \\
Lago & 19,0 \\
É afastado da cidade & 10,5 \\
Os animais silvestres & 7,2 \\
As pessoas & 3,3 \\
Outros & 1,9 \\
\hline
\end{tabular}

(Base de dados referente aos 363 entrevistados [60,5\%] que visitaram o Parque Quedas do Rio Bonito, independentemente de sua freqüência).

\section{DISCUSSÃO}

O Parque Quedas do Rio Bonito possui sua importância, não só para a população de média e alta renda da área urbana, mas também como atração turística da região. Mas, é inevitável que a cidade de Lavras possua, dentro do perímetro urbano, um parque com dimensões mais signifi- cativas e que ofereça à população atividades de lazer e estrutura física adequada, de modo a oferecer lazer ativo e passivo a todas as idades e classes de renda.

Com o passar dos anos, com o crescimento da cidade, locais que atualmente são considerados como áreas verdes, como, por exemplo, as praças da cidade com área superior a $200 \mathrm{~m}^{2}$, com predominância de elementos vegetativos, já não irão suprir as necessidades da população.

Se não houver uma política de continuidade que vise à proteção e manutenção das áreas verdes públicas urbanas existentes e à criação de novas áreas, poderá ocasionar uma queda considerável na qualidade de vida da população lavrense.

\section{CONCLUSÃO}

a) O Parque Quedas do Rio Bonito é freqüentado por $60,5 \%$ dos entrevistados, dos quais $3 \%$ desconheciam a sua existência.

b) Dos que nunca foram ao parque, $38,4 \%$ pertencem à classe de baixa renda; $50,2 \%$ pertencem à classe de renda média e $11,4 \%$ pertencem à classe de renda alta.

c) Os frequientadores do parque pertencem às classes de renda alta $(30,6 \%)$ e média $(53,2 \%)$.

d) Os principais motivos alegados pelos entrevistados por nunca terem visitado o parque foram: a falta de condução $(40,2 \%)$, falta de oportunidade $(34,7 \%)$, falta de motivação/interesse $(21,9 \%)$, ter que pagar ingresso $(16,4 \%)$.

e) As atividades de lazer que mais agradam os entrevistados são: contato com a natureza $(74,7 \%)$, paisagem $(60,6 \%)$, cachoeira $(51,2 \%)$, trilha $(39,9)$ e vegetação $(21,5 \%)$.

\section{REFERÊNCIAS BIBLIOGRÁFICAS}

BEARZOTI, E.; OLIVEIRA, M. S. Estatística básica. Lavras: UFLA, 1997. $191 \mathrm{p}$.

DEMATTÊ, M. E. S. P. Princípios de paisagismo. 2. ed. Jaboticabal: Funep, 1999. 101 p.

MARCONI, M. A. Técnicas de pesquisa: planejamento e execução de pesquisas, amostragens e técnicas de pesquisas, elaboração, análise e interpretação de dados. 3. ed. São Paulo: Atlas, 1996. 231 p.

UNIVERSIDADE FEDERAL DE LAVRAS. Lavras/MG: turismo: parque florestal Quedas do Rio Bo-

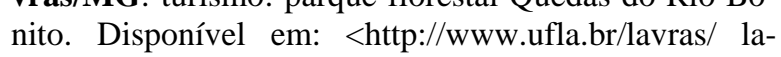
vras 1/parque.htm>. Acesso em: 24 nov. 2002 . 